\title{
Investigation of photoluminescence and amplified spontaneous emission properties of cyanoacetic acid derivative (KTB) in PVK amorphous thin films
}

\author{
Julija Pervenecka*a, Aivars Vembris ${ }^{\mathrm{a}}$, Elmars Zarins ${ }^{\mathrm{b}}$, Valdis Kokars ${ }^{\mathrm{b}}$ \\ anstitute of Solid State Physics, University of Latvia, 8 Kengaraga Str., Riga LV-1063, Latvia; \\ 'Institute of Applied Chemistry, Riga Technical University, 14/24 Azenes Str., Riga LV-1048, \\ Latvia
}

\begin{abstract}
In this work photoluminescence and amplified spontaneous emission properties of new original 2-cyanoacetic acid derivative mixed in polyvinyl carbazole (PVK) matrix at different concentration were investigated. Ethyl 2-(2-(4-(bis(2(trityloxy)ethyl)amino)styryl)-6-tert-butyl-4H-pyran-4-ylidene)-2-cyanoacetate (KTB) is recently synthesised nonsymmetric red light emitting laser dye, that in previous experiments with neat thin films showed low amplified spontaneous emission (ASE) threshold value. Based on PVK high refractive index it has been used as a polymer to ensure good planar waveguide structure.

According to the previous experiments luminescence quenching is expected in neat amorphous thin films which reduces photoluminescence quantum yield and increases ASE excitation threshold energy. It could be overcome by doping the laser active molecules in polymer matrix.

The lowest threshold value of ASE was achieved at 20wt $\%$ of KTB molecules in PVK matrix. Ability to significantly decrease intermolecular interactions and excitation threshold energy of investigated compound in the host-guest systems makes KTB promising to be used as a laser dye in preparation of organic solid state lasers.
\end{abstract}

Keywords: cyanoacetic acid derivative, glass forming low molecular weight compounds, amplified spontaneous emission, laser dyes, guest-host system

\section{INTRODUCTION}

One of the technology field in which light-emitting materials could be used is associated with organic solid-state lasers ${ }^{1}$. Organic solid-state lasers are in over the past decades actively developing technology in which different non crystalline structure films are widely used ${ }^{1,2,3}$. Optically pumped lasing action in thin films of light-emitting molecules makes organic solid-state laser competitive with more expensive traditional inorganic lasers, due to much cheaper fabrication processes, small sizes, easily processable and easily integrated into photonic devices. The main requirement to use lightemitting materials in lasers is high light amplification coefficient, low ASE excitation threshold energy $\left(\mathrm{few} \mu \mathrm{J} / \mathrm{cm}^{2}\right)$ and the possibility to form non-crystallized and no aggregates containing structure.

The main obstacles to reach low amplified spontaneous emission (ASE) excitation threshold value are intermolecular interactions between dye molecules. The first attempts to overcome started with synthesize molecules with attached large bulky groups to a chromophore which don't contribute to light emission properties of the molecule. Inspired by the significant light emitting properties of well-known laser dye DCM, it was decided to start exactly with $i^{4}$. The first attempts were unsuccessful. The main problem was a high intermolecular interaction between DCM dye molecules which occurs in the thin films. It results in luminescence quenching which reduces photoluminescence quantum yield and extremely increases ASE excitation threshold energy. Intermolecular interaction was varied by modified DCM dye with different substituents in the electron donor part. Modification of DCM dye by substituted one of the methyl in the electron donor part with the bulky trityloxyethyl groups results in DWK-14, ${ }^{4}$, replacement with the tert-butyl substituent (DWK-1TB $)^{6}$, by substitution of the two of the methyl in the electron donor part - DWK-2, 5 , etc. Such attempts were tempted on decreasing intermolecular interactions between the dye molecules by increasing a distance between the molecules due to increasing spatial size of the molecules themselves without changing their light emitting properties. 
The contribution of attached bulky groups was good as expected - ASE excitation threshold energy value decreased, but such decreased excitation energy was not too low, to compete with already used laser dyes.

The second step to reach aim was the preparation of dyes in polymer host-guest system where was achieved decreases of intermolecular interactions between dye molecules. Many varieties of different polymers were investigated, but in among the best were only two: $\mathrm{PMMA}^{7}$ and $\mathrm{PVK}^{8}$. Such systems provided many varieties of concentration proportional between host and guest compounds. Despite of wide range of investigated weight ratios of dye molecules:polymer system, experimentally was proved that for each dye molecule exist narrow weight ratio of prepared dye molecules:polymer host-guest system at which compounds ASE excitation threshold energy is the lowest. For example: for well-known laser dye DCM the lowest excitation threshold energy could be obtained within the concentration range between 2 and $4 \mathrm{wt} \%^{7,8}$, but for its promising symmetric and non-symmetric derivatives DWK- ${ }^{8}$, DWK- $2^{8}$ and DWK$1 \mathrm{~TB}^{8}$ it could be obtained within the concentration range between 15 and $20 \mathrm{wt} \%{ }^{8}$.

In this paper were investigated new original non-symmetric 2-cyanoacetic acid derivative ethyl 2-(2-(4-(bis(2(trityloxy)ethyl)amino)styryl)-6-tert-butyl-4H-pyran-4-ylidene)-2-cyanoacetate (KTB) in polyvinylcarbazole (PVK). Optical and ASE properties of the different concentration host-guests systems were investigated.

\section{MATERIALS AND METHODS}

\subsection{Investigated organic compounds}

Ethyl 2-(2-(4-(bis(2-(trityloxy)ethyl)amino)styryl)-6-tert-butyl-4H-pyran-4-ylidene)-2-cyanoacetate (KTB) was synthesized from ethyl 2-(2-tert-butyl-6-methyl-4H-pyran-4-ylidene)-2-cyanoacetate and 4-(bis(2(trityloxy)ethyl)amino)benzaldehyde. Investigated KTB molecule consists of 4-substituted-4H-pyrane as an electron acceptor and one an electron donor group with attached two bulky trityloxyethyl groups at the electron donor side (see Fig.1). Bulky trityloxyethyl groups assist to prepare amorphous thin films from solution and weaken intermolecular interaction which in turn reduces crystallization.<smiles>CCOC(=O)[C@@H](C#N)c1cc(/C=C/c2ccc(N(CCOC(c3ccccc3)(c3ccccc3)c3ccccc3)CCOC(c3ccccc3)(c3ccccc3)c3ccccc3)cc2)c(C(C)(C)C)o1</smiles>

Figure 1. Chemical structure of KTB.

\subsection{Sample preparation for optical characterization}

Firstly, pure KTB was dissolved in dichloromethane. Then, to obtain a guest-host system, the necessary amount of the prepared KTB-dichloromethane solution was added to PVK (Sigma Aldrich No. 368350) with KTB dye concentration of $1,5,10,20,30,50,70 \mathrm{wt} \%$. Organic compound mass to the solvent volume ratio was kept constant (30 mg/ml) for all the prepared solutions. A constant ratio of the organic compound and solvent is necessary to provide a similar film thickness of all the samples. Before the deposition, glass substrates were cleaned in dichloromethane and for $10 \mathrm{~min}$ heated at $115^{\circ} \mathrm{C}$. Thin films were deposited on the substrate by the spin-coating technique. The solutions were spincoated on the substrates for $40 \mathrm{~s}$ at spin speed $800 \mathrm{rpm}$ and acceleration of $800 \mathrm{rpm} / \mathrm{s}$ and then dried at $85^{\circ} \mathrm{C}$ for $10 \mathrm{~min}$. The thickness of all the films was about $300 \mathrm{~nm}$ which satisfied waveguide conditions. 


\subsection{Measurement systems}

Absorption spectra of the thin films were measured by an Ocean Optics HR4000 spectrometer.

Photoluminescence spectra and photoluminescence quantum yield (PLQY) were measured by the calibrated system Fluorescence spectrometer Pico Master 1 (Photo Med GmbH). It consists of xenon lamp - monochromator - integrated sphere - monochromator - detector. Spectral resolution was set to $2 \mathrm{~nm}$. The excitation wavelength was chosen at the KTB absorption maximum: $471 \mathrm{~nm}$. Firstly excitation and emission spectrum were obtained when only glass slide was in the sphere. After which excitation and emission spectrum were recorded when the thin film was in the sphere. All four spectra were used for PLQY calculation.

Setup for ASE measurement is self-made. Its experimental scheme is shown in Fig.2. Ekspla 310 series pulse laser with tunable wavelength was used as the excitation source. Amplified spontaneous emission was excited at $471 \mathrm{~nm}$ wavelength. Pulse duration was $10 \mathrm{~ns}$ and repetition rate $10 \mathrm{~Hz}$. The pulse energy was tuned by Glan prism/half wave plate/Glan prism system. Beam expander was used to extend beam 5 times to $25 \mathrm{~mm}$. The beam was focused by a cylindrical lens to $0.4 \mathrm{~mm}$ wide line on the sample surface. The slit was used to cut-off edges of the beam and get $3 \mathrm{~mm}$ long line with equal intensity on the sample. Beam intensity before the sample was measured by the power meter. The emitted light was collected from the edge of the sample by optical fibre, which, going through long pass filter with a cut on wavelength till $500 \mathrm{~nm}$, was connected to the calibrated Ocean Optics spectrometer HR 4000.

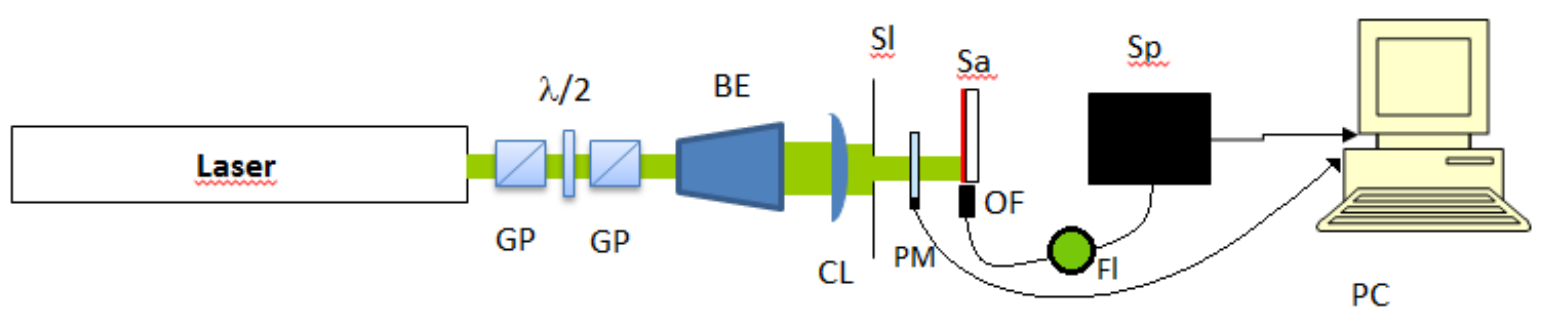

Figure 2. Experimental scheme of amplified spontaneous emission measurement. GP - Glan prism, $\lambda / 2$ - half wave plate, $\mathrm{BE}$-beam expender, $\mathrm{CL}$ - cylindrical lens, $\mathrm{Sl}$ - slit, $\mathrm{PM}$ - power meter, $\mathrm{Sa}$ - samples, $\mathrm{OF}$ - optical fibre, $\mathrm{Fl}$ - long pass filter and $\mathrm{Sp}$ - spectrometer Ocean Optics HR4000.

\section{RESULTS AND DISCUSSION}

\subsection{Optical images}

Optical images of the surface morphological structure of the samples (see Fig.3) were obtained by high resolution optical microscope Nikon ECLIPSE L150. At all optical images of the prepared thin films surface have been observed small dots. Looking at the nature of the origin of these dots, the formation of crystals or residues of an insoluble substance could be excluded. In the opposite case, the thickness of the layer at these locations would be greater, which could be observed as interfering rings around them or diffraction of light in the crystals themselves. Most probably these dots are bubbles of the uneven evaporation of the solvent, formed due to the non-response of the spin speed to an increase in the viscosity of the solution due to the high concentrations of the polymer in it. Bubbles "frozen" in thin films structure do not affect films optical properties and quality of further measurements. In pure thin films of KTB, such bubbles or some other inhomogeneities in the layer have not been observed. 

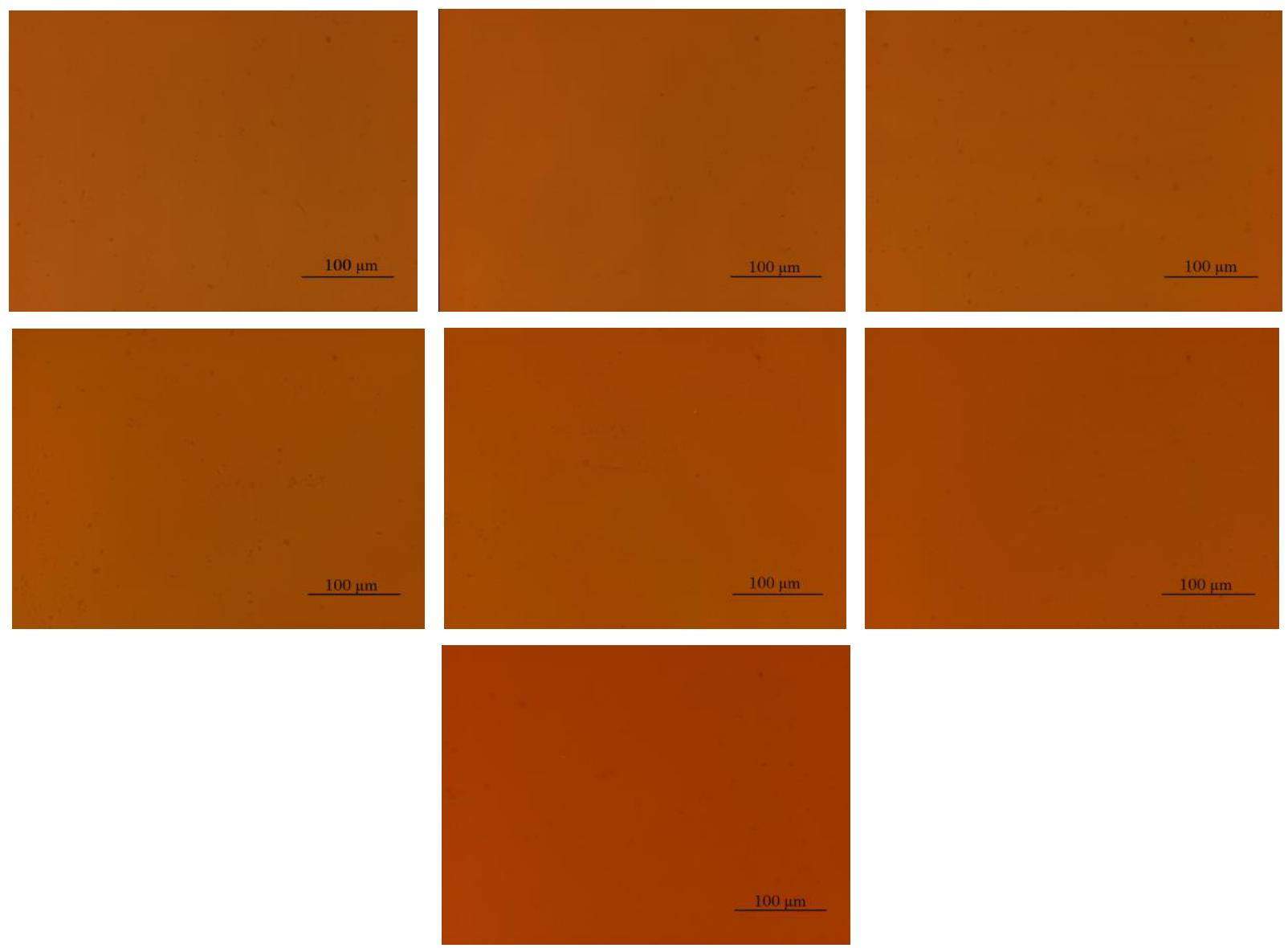

Figure 3. High resolution optical images of the samples surface morphology. Compound concentration in the PVK matrix is given on the images.

\subsection{Optical properties}

Absorption spectra of all studied KTB:PVK systems practically do not differ depending on KTB dye concentration in the PVK matrix. Absorption spectra of KTB $20 \mathrm{wt} \%$ is shown in Fig. 4.

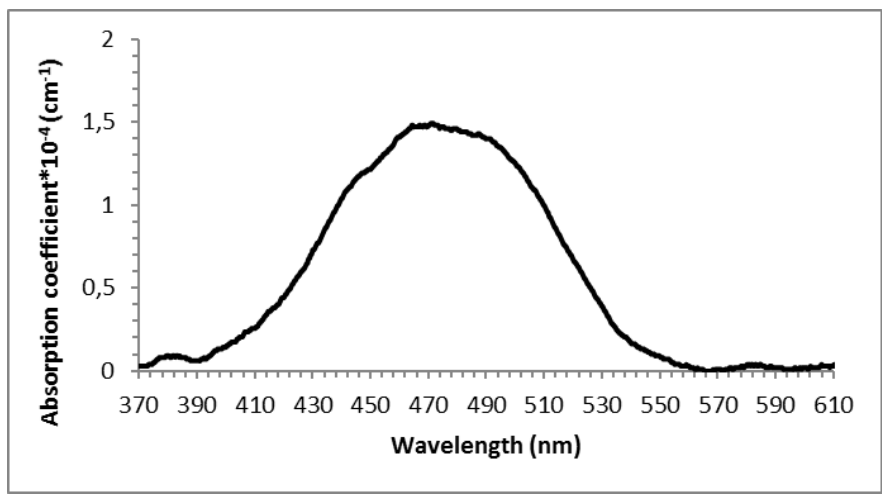

Figure 4. Absorption spectra of $20 \mathrm{wt} \%$ KTB in PVK matrix.

Photoluminescence quantum yield decreases at higher KTB concentration (see Fig.5a) which explains by decreases of a distance between the molecules due to increases of the dye concentration in the system. 


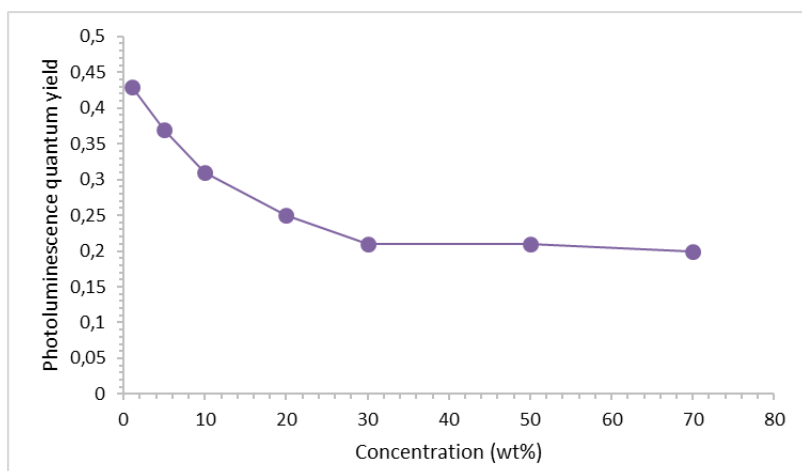

a)

Figure 5. a) Dependence of photoluminescence quantum yield on dye concentration in PVK matrix; b) Photoluminescence maximum position at different dye concentrations.

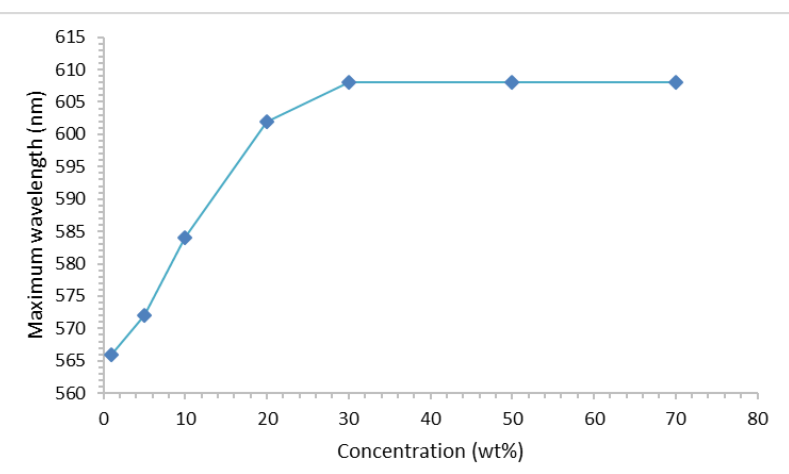

b)

Photoluminescence (PL) spectra of KTB:PVK has small red shifts with the increases of KTB dye concentration in PVK matrix (see Fig.6). Such shifting of photoluminescence spectra is called solid state solvation effect ${ }^{7,8}$ it already has been observed in different host-guest systems ${ }^{7,8,9,10}$. It is a usual phenomenon in solutions but relatively rarely has been observed in solid states systems. In solutions, it is known as a solvatochromic effect which appears due to different polarities of used solutions. The explanation of spectral shift in the solid state is related to changes of "solid liquid" dielectric constant due to increases of guest dye concentration in the host (polymer).

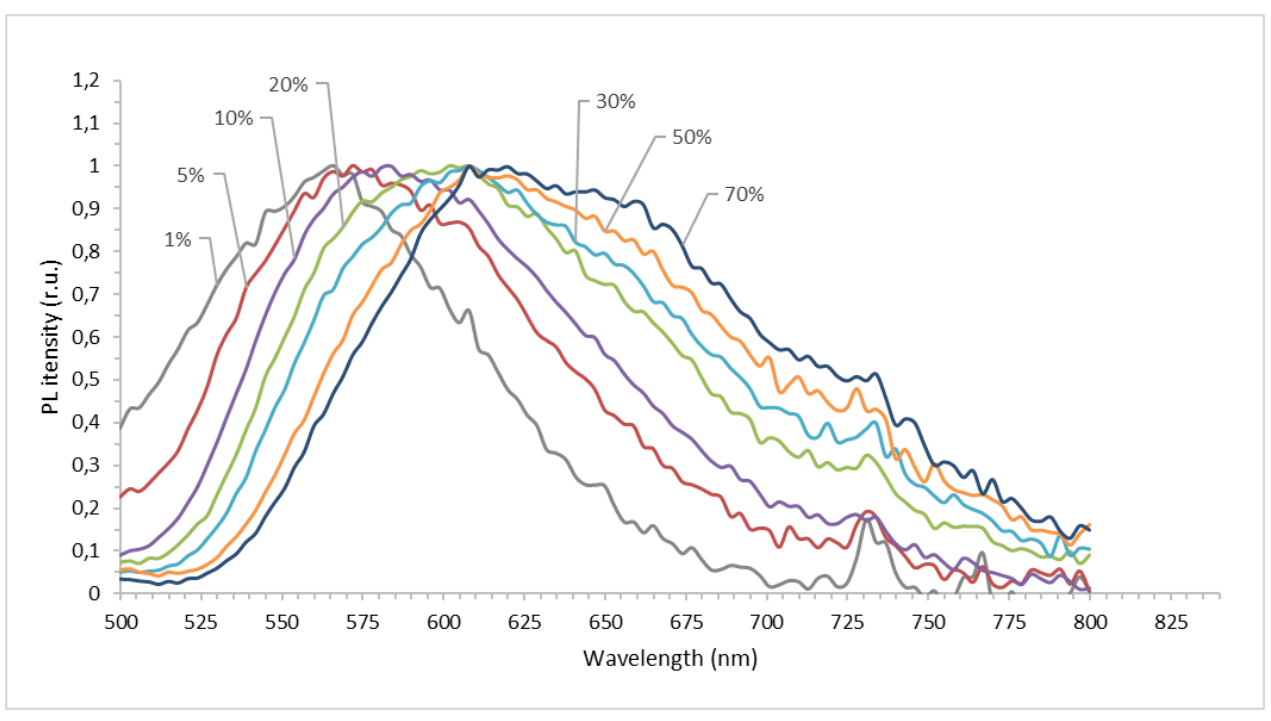

Figure 6. Photoluminescence spectra at different KTB concentrations in PVK matrix

\subsection{Amplified spontaneous emission}

An optical quality of all samples was good enough, as could be seen from optical images (see Fig.3), no noticeable defects on the surface have not been observed. For amplified spontaneous emission excitation (ASE) for all the samples were used the same wavelength of $471 \mathrm{~nm}$, that appropriates to KTB absorption maximum wavelength (see Fig.4). The amplified spontaneous emission of excited thin films has been observed as the appearance of the narrow high intense peak on top of PL band (see Fig.7). The full width of half maximum (FWHM) of emission spectrum decreases by around one order of magnitude with the increases of pump beam energy. 


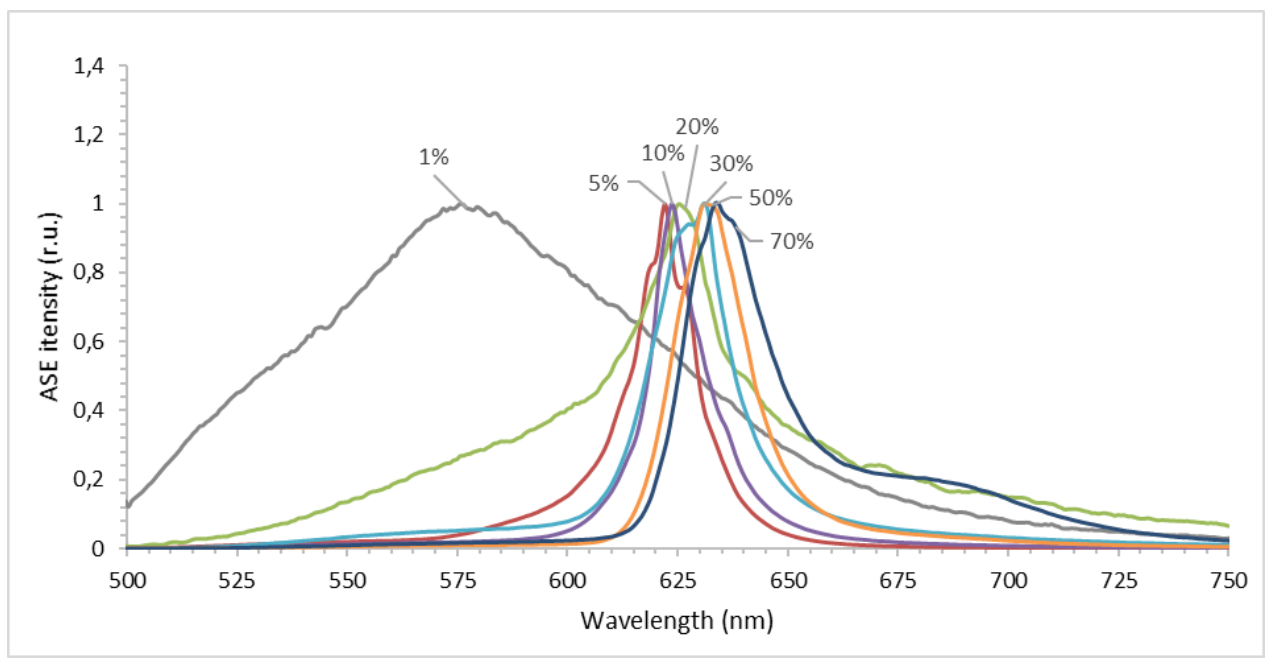

Figure 7. ASE spectra at different KTB dye concentrations in PVK matrix.

ASE emission spectra of each investigated host-guest system are slightly red shifted from PL spectra. Such shifting is explained by theoretical statement according to which cross section of stimulated emission should be larger on the right side of PL spectral band maximum ${ }^{6}$. Similar to PL, have been observed less expressed redshifts of ASE spectra from lower to higher wavelengths, which depending on dye molecule concentration in the polymer matrix. Such shifts are related to overlapping of PL and absorption spectral bands. In this case, the formation of ASE peak depends on both absorption coefficient and optical gain. ASE peak could forms only on those PL spectral band parts, where optical gain is bigger than absorption coefficient ${ }^{11}$. The area of the photoluminescence and absorption spectral bands overlap decreases with increasing concentration of the dye molecule in the polymer matrix. The influence of absorption increases with the size of the overlap. Therefore, the ASE spectra are less redshifted at lower weight ratios of the dye molecule in the polymer matrix than at higher (see Fig.8b).
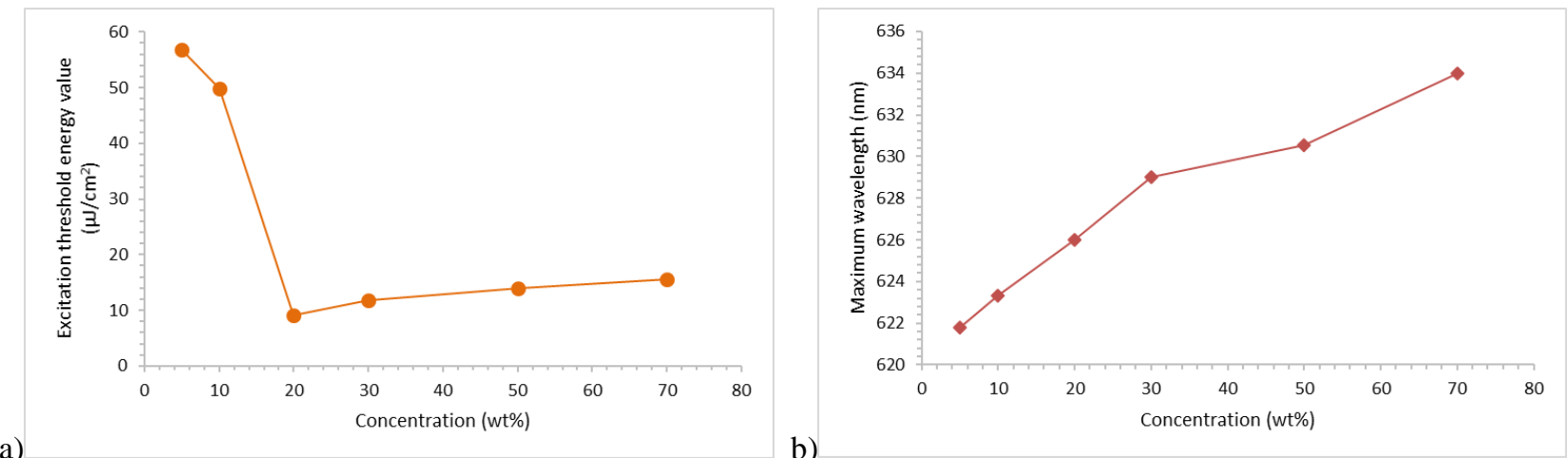

a)

b)

Figure 8. Dependence of: a) Excitation threshold energy value on dye concentration; b) ASE maximum wavelength on dye concentration.

Excitation threshold energy value decreases from $5-20 \mathrm{wt} \%$ by reaching a minimum at $20 \mathrm{wt} \%$, then again increases with increasing dye molecule concentration (see Fig.8a). The best result of ASE excitation threshold energy value (9 $\mu \mathrm{J} / \mathrm{cm}^{2}$ ) has been reached at $20 \mathrm{wt} \%$ of KTB in PVK matrix. That makes guest-host systems of KTB:PVK perspective to be used in the preparation of laser active medium. 
Table 1.

Optical properties of KTB at different concentrations

\begin{tabular}{r|r|r|r|r|r}
\hline $\begin{array}{c}\text { Concentration, } \\
\mathbf{w t} \%\end{array}$ & $\lambda_{\text {ABS, }} \mathbf{n m}$ & $\lambda_{\text {PL }}, \mathbf{n m}$ & $\lambda_{\text {ASE }}, \mathbf{n m}$ & PQLY, $\%$ & $\mathbf{E}_{\text {th }, \boldsymbol{\mu J} / \mathbf{c m}^{2}}$ \\
\hline 1 & 471 & 566 & - & 43 & - \\
\hline 5 & 471 & 572 & 622 & 37 & 57 \\
\hline 10 & 471 & 584 & 623 & 31 & 50 \\
\hline 20 & 471 & 602 & 626 & 25 & 9 \\
\hline 30 & 471 & 608 & 629 & 21 & 12 \\
\hline 50 & 471 & 608 & 631 & 21 & 14 \\
\hline 70 & 471 & 608 & 634 & 20 & 16 \\
\hline$\Delta$ & \pm 2 & \pm 2 & \pm 2 & \pm 2 & \pm 10 \\
\hline
\end{tabular}

\section{CONCLUSIONS}

Photoluminescence quantum yield decreases at the higher KTB concentration. KTB:PVK systems photoluminescence and amplified spontaneous emission spectra could be tuned by dye molecule concentration in the polymer matrix due to solid state solvation effect dependence. The lowest ASE excitation threshold energy value $9 \mu \mathrm{J} / \mathrm{cm}^{2}$ was obtained at 20 wt\% of KTB in PVK matrix.

\section{ACKNOWLEDGEMENT}

This work was supported by European Regional Development Fund within the Project No. Nr.1.1.1.1/16/A/046 and A.Riekstina SIA “Mikrotīkls" donation, administered by University of Latvia Foundation.

\section{REFERENCES}

[1] Forget, S., Chenais, S, [Organic Solid-State Lasers], Springer, Heidelberg \& New York 175, 1-179 (2013).

[2] Tessler, N., "Lasers based on semiconducting organic materials," Adv. Mater. 11, 363-370 (1999).

[3] Kozlov, V.G., Forrest, S.R., "Lasing action in organic semiconductor thin films," Curr. Opin. Solid State Mater. Sci. 4, 203-208 (1999).

[4] Vembris, A., Muzikante, I., Karpicz, R., Sliauzys, G., Miasojedovas, A., Jursenas, S., Gulbinas, V., "Fluorescence and amplified spontaneous emission of glass forming compounds containing styryl-4H-pyran-4-ylidene fragment," Journal of Luminescence 132, 2421-2426 (2012).

[5] Zarins, E., Jubels, J., Kokars, V., "Synthesis of red luminescent non symmetric styryl-4H-pyran-4-ylidene fragment containing derivatives for organic light-emitting diodes," Advanced Materials Research 222, 271-274 (2011).

[6] Zarins, E., Vembris, A., Misina,E., Narels, M., Grzibovskis, R., Kokars, V., "Solution processable 2-(trityloxy)ethyl and tert-butyl group containing amorphous molecular glasses of pyranylidene derivatives with lightemitting and amplified spontaneous emission properties," Optical Materials 49, 129-137 (2015).

[7] Vembris, A., Zarins, E., Kokars, V., "Solid state solvation effect and reduced amplified spontaneous emission threshold value of glass forming DCM derivative in PMMA films," Journal of Luminescence 158, 441-446 (2015).

[8] Vembris, A., Zarins, E., Kokars, V., "Stimulated emission and optical properties of pyranyliden fragment containing compounds in PVK matrix," Optics and Laser Technology 95, 74-80 (2017).

[9] Bulovic, V., Shoustikov, A., Baldo, M., Bose, E., Kozlov, V.G., Thompson, M.E., Forrest, S.R., "Bright, saturated, red-to-yellow organic light-emitting devices based on polarization-induced spectral shifts," Chemical Physics Letters 287, 455-460 (1998).

[10] Bulovic, V. R., Thompson, E., Forrest, S.R., "Tuning the color emission of thin film molecular organic light emitting devices by the solid state solvation effect," Chemical Physics Letters 308, 317-322 (1999). 
[11] Popova, S., Pudzs, K., Latvels, J., Vembris, A., "Light emitting and electrical properties of pure amorphous thin films of organic compounds containing 2-tert-butyl-6-methyl-4H-pyran-4-5 ylidene," Optical Materials 36(2), 529534 (2013).

Institute of Solid State Physics, University of Latvia as the Center of Excellence has received funding from the European Union's Horizon 2020 Framework Programme H2020-WIDESPREAD-01-2016-2017TeamingPhase2 under grant agreement No. 739508, project CAMART ${ }^{2}$ 